\title{
Sensor Selection Scheme considering Uncertainty Disturbance
}

\author{
Shi Wentao $\mathbb{D}^{1}$ Chen Dong, ${ }^{1}$ Zhou Lin $\mathbb{D},{ }^{2}$ Bai Ke, ${ }^{2}$ and Jin Yong $\mathbb{D}^{2}$ \\ ${ }^{1}$ School of Marine Science and Technology, Northwestern Polytechnical University, Xi'an 710072, China \\ ${ }^{2}$ School of Artificial Intelligence, Henan University, Zhengzhou 450046, China
}

Correspondence should be addressed to Zhou Lin; zhoulin@henu.edu.cn

Received 27 October 2021; Accepted 18 December 2021; Published 16 February 2022

Academic Editor: Xue-bo Jin

Copyright (c) 2022 Shi Wentao et al. This is an open access article distributed under the Creative Commons Attribution License, which permits unrestricted use, distribution, and reproduction in any medium, provided the original work is properly cited.

In multisensor cooperative detection network, some random disturbances, energy carried by sensor, distance between target and sensor node, and so on all affect the sensor selection scheme. To effectively select some sensors for detecting the target, a novel sensor selection method considering uncertainty disturbance is proposed under constraints of estimation accuracy and energy consumption. Firstly, the sensor selection problem is modeled as a binary form optimization problem with a penalty term to minimize the number of sensors. Secondly, some factors (precision, energy, and distance, etc.) affecting the sensor selection scheme are analyzed and quantified, and energy consumption matrix and estimation precision threshold are given by matrix tra'nsformation. Finally, the problem of minimizing sensor number after relaxation is solved by convex optimization method, obtaining sensor selection scheme by discretization and legitimization of the suboptimal solution after convex relaxation. Simulation results show that the proposed algorithm can ensure the minimum number of sensors, improving accuracy of state estimation and saving network energy.

\section{Introduction}

Wireless Sensor Network (WSN) is a self-organization wireless network composed of large numbers of microsensor nodes. Due to it being easy to deploy and inexpensive, WSN widely is applied in areas such as the military, aviation, and agriculture [1]. At the same time, all sensor nodes in WSN can be used to obtain the most comprehensive information related to target; however, it leads to the energy consumption and processing burden of the network. Thus, according to measurement accuracy requirements, selecting part sensor nodes can save network energy, reduce response delay, and prolong the lifetime of the network [2].

1.1. Related Works. Some researches of sensors selection have been applied in target tracking system, cooperative detection system, and so on [3,4]. Jamali et al. embedded a penalty item into objective function of sensors selection to enhance sparsity of solver, and it was only used in low dimension scene [5]. A greedy algorithm called FrameSense was proposed by Ranieri et al., and it iteratively removed the sensor corresponding to the maximum cost function [6]. FrameSense's algorithm could obtain optimal sensor selection scheme, but heavy compute burden and multiple constraint conditions were needed. Minimum eigenspace method was proposed by Jiang et al., which could quickly converge to obtain sensor selection scheme; however, lower estimation accuracy was its disadvantage [7], so an improved minimum eigenspace method with group greedy scheme was proposed by Jiang et al. to improve about $63 \%$ estimation accuracy expect heavy compute burden [8].

In spite of sensor selection can be modeled as integer programming, it is NP-hard problem. Branch and bound (BBD) was an effective global optimal method corresponding to integer programming; however, computation complexity was exponentially growing with increase of dimension corresponding to optimized variable [9]. Based on convex method, Joshi and Boyd proposed a heuristic method to approximately obtain sensor selection scheme [10]. Since the constraint of the problem was relaxed, Joshi and Boyd's method could reduce the accuracy of measurement. So, some local optimum techniques were proposed by Joshi to enhance accuracy corresponding to sensor 
selection problem, while it had good detection performance with a small number of sensors.

Besides, communication performance and remaining energy of sensors are two key factors in multisensor cooperative detection network, and they should be considered in sensor selection too. Sensor selection technique with time, energy, and communication constraints was discussed by Rusu et al. [11]; they modeled the sensor selection problem as a $0-1$ optimization problem, then used a convex relaxation method to solve the objective function. However, the influence of some factors (sensor measurement capability, sensor location, etc.) on sensor scheduling was not involved in Rusu et al.'s paper. In addition, some various factors (uncertainty disturbance, vibration disturbance, etc.) caused by complexity environment may decrease measurement accuracy of selected sensors. So, it is important that selecting suitable sensors under some constraints including measurement accuracy, energy consumption and communication capability.

1.2. Contributions. Under some conditions including bounded disturbance of sensor measurement and sensor location, a new sensor selection algorithm based on the constraints of energy and estimation accuracy is proposed. By using convex relaxation technology, the minimum number of sensor selection set is obtained, and it balances network energy and measurement accuracy to ensure the performance of the multisensor cooperative detection system. The main contributions are as follows:

(1) A binary form optimization model with penalty term is proposed to obtain the sensor selection scheme, which is used to minimize number of sensors. Comparing with tradition algorithms, our energy penalty term is used to balance energy consumption

(2) The distance weight between sensor and target is adaptively given. It directly reflects that the closer the sensor is to target, the greater probability of being selected, and the better detection accuracy of sensor. So, compared with the traditional method, the number of selected sensors can be effectively reduced

(3) The energy consumption matrix and state estimation precision are regarded as constraints of the sensor selection problem; it more reflects various factors of the complex environment

(4) We introduce techniques including the convex relaxation, discretization, and legitimization of bounded interval $[0,1]$, which are used to handle Np-hard problem of sensor scheduling and receive suboptimal solution. The proposed method has lower computation complexity than traditional methods

1.3. Paper Organization and Notation. The rest of this paper is organized as follows. Section 2 is problem statement for knowledge research background. Section 3 proposes the improved method for the sensor selection scheme under various constraints including precision, energy, and distance. In this section, sensor selection with penalty term, sensor selection considering uncertainty disturbance, optimization of sensor selection, and description of the proposed algorithm are given separately. Section 4 shows simulation results and analysis. Some conclusions about this paper are given in Section 5.

The symbol denotement of this paper is descripted as shown in Table 1.

\section{Problem Statement}

Consider a target $\mathbf{x}$ with $\mathrm{N}$-dimensional state is cooperatively detected by sensor set $\Omega$ which composed of $M$ sensors. Measurement matrix $\Phi$ is related to set $\Omega$, which can be defined as $\Phi=\left[\varphi_{1}, \varphi_{2}, \cdots, \varphi_{M}\right]^{T} \in R^{M \times N}, M>N$, and $\varphi_{i} \epsilon$ $R^{N \times 1}$ is the measurement vector corresponding to sensor $i$. At a sampling time $t, k_{t}$ sensors are selected to form measurement sensor set $\Omega_{k t}\left(\Omega_{k t} \subseteq \Omega\right)$; at the same time, measurement of target state $\mathbf{x}_{t}$ is described as follows:

$$
\mathbf{y}_{t}=\Phi_{t} \mathbf{x}_{t}+v_{t}
$$

where $\Phi_{t} \in R^{k_{t} \times N}, k_{t}>N$ is the measurement matrix and $\mathbf{y}_{t}$ $\in \mathbf{R}^{k_{t}}$ is the measurement of sensors.

Therefore, the minimum unbiased estimate of target state $\mathbf{x}_{t}$ is $\widehat{\mathbf{x}}_{t}=\Phi_{t}^{\dagger} \mathbf{y}_{t}=\left(\Phi_{t}^{T} \Phi_{t}\right)^{-1} \Phi_{t}^{T} \mathbf{y}_{t}$, and its mean square error (MSE) can be expressed as [12]

$$
\operatorname{MSE}\left(\widehat{\mathbf{x}}_{t}\right)=\operatorname{tr}\left(\left(\Phi_{t}^{T} \Phi_{t}\right)^{-1}\right)=\sum_{i=1}^{n} \frac{1}{\lambda_{i}\left(\Phi_{t}^{T} \Phi_{t}\right)} .
$$

\section{The Proposed Algorithm for Sensor Selection}

To solve the problem of sensor selection under some constraints, the optimization problem of sensor selection is given under some constraints including environment, energy, and distance between node and target. After being quantized, constraints can be described with the energy consumption matrix and state estimation precision to be regarded as constraints of the optimization problem. Besides, the distance weight between the sensor and target is adaptively given to reflect that the closer the sensor is to target, the greater the probability of being selected. Convex relaxation methods are considered to handle optimization problem of sensor selection, and discretization and legitimization of suboptimal solution after convex relaxation are used to obtain the suboptimal solution of the sensor selection scheme.

3.1. Problem of Sensor Selection. Obviously, state estimation accuracy of target $\mathbf{x}$ is the highest $\gamma_{0}=\operatorname{tr}\left(\left(\Phi^{T} \Phi\right)^{-1}\right)$ when sensor set $\Omega_{k t}=\Omega$, i.e., all sensors of sensor cooperative detection system are used to detect target. However, it consumes a lot of energy; moreover, there are some uncertain environment factors such as temperature, humidity, and visibility, which reduce the estimation accuracy of the target state. Therefore, considering environment factors and estimation performance, the problem of sensor selection can 
TABLE 1: Symbol denotement.

\begin{tabular}{lc}
\hline Symbol & Denotement \\
\hline$\Phi($ or $v)$ & Measurement matrix (vector) \\
$(\cdot)_{i}$ & $i$-th element of vector \\
$\mathbf{1}$ & Vector with proper dimension whose entry is 1 \\
$\operatorname{diag}(\cdot)$ & Diagonal of matrix \\
$\operatorname{card}(\cdot)$ & Cardinal of given set \\
$\lambda_{i}(\cdot)$ & $i$-th eigenvalue of matrix \\
$(\cdot)^{T}$ & Transposition of matrix \\
$(\cdot)^{-1}$ & Inverse of matrix \\
$\operatorname{tr}(\cdot)$ & Trace of matrix \\
$\odot$ & Hadamard product \\
$\|\cdot\|_{2}$ & 2-Norm \\
$\|\cdot\|_{\mathbf{F}}$ & F-Norm \\
$\|\cdot\|_{\infty}$ & $\infty$-Norm \\
$t$ & Sampling time \\
$\mathbf{x}_{t}$ & Cero mean Gaussian noise with $\sigma^{2} \mathbf{I}$ covariance \\
$k_{t}$ & Measurement of sensor \\
$\Phi_{t}$ & Coordinate of target $\mathbf{x}$ \\
$v_{t}$ & Number of sensors \\
$\mathbf{y}_{t}$ & \\
$\mathbf{b}$ & \\
\hline
\end{tabular}

be expressed as the minimum cardinal of sensor measurement set $\Omega_{k t}$ as follows:

$$
\begin{array}{cc}
\underset{\Omega_{k t}}{\operatorname{minimize}} & \sum_{t=1}^{T} \operatorname{card}\left(\Omega_{k t}\right), \\
\text { s.t. } & r\left(\left(\Phi_{t}^{T} \Phi_{t}\right)^{-1}\right) \leq \tau \gamma_{0}-C, \\
& C-\gamma_{0}(\tau-1) \leq 0,
\end{array}
$$

where card $(\cdot)$ is the function of minimum cardinal. $T$ is the total sampling time. $\tau>1$ is the accuracy level which increases with the decrease of accuracy requirement. $C=V /\left(p h t_{0}\right)(V$, $p, h, t_{0}$ express visibility, environmental factor parameter, humidity, and temperature, respectively) is the environment factor. When $\tau$ increases, it means that fewer sensors can be used to meet estimated accuracy requirement; besides, inequality $C-\gamma_{0}(\tau-1) \leq 0$ is used to restrict the estimation accuracy which does not exceed the optimal estimation accuracy.

In short, equation (3) indicates that we want to choose a sensor set with minimum cardinal satisfying estimation accuracy constraint conditions. To describe the problem of sensor selection, $M$-dimensional vector $\omega_{t} \in\{0,1\}^{M}$ is introduced to express the selected state of sensors in sensor set $\Omega$ at $t$ sampling time, and $\left(\omega_{t}\right)_{i}$ encodes whether the $i$-th sensor is selected. The selected state of sensors at all sampling time $T$ can be described by matrix $\Theta=\left[\omega_{1}, \omega_{2}, \cdots, \omega_{T}\right]$. Thus, the optimization problem of sensor selection described in equation (3) can be transformed into the following equation:

$$
\begin{array}{cc}
\underset{\Theta \in\{0,1\}^{M \times T}}{\operatorname{minimize}} & \sum_{t=1}^{T} \alpha_{t}^{T} \omega_{t}, \\
\text { s.t. } \quad \operatorname{tr}\left(\left(\Phi^{T} \operatorname{diag}\left(\omega_{t}\right) \Phi\right)^{-1}\right) \leq \tau \gamma_{0}-C, \\
C-\gamma_{0}(\tau-1) \leq 0,
\end{array}
$$

where weight vector $\alpha_{t} \in R^{M}$ and $\left(\alpha_{t}\right)_{i}=\left(\left(\omega_{t}\right)_{i}+\epsilon\right)^{-1}$ $(i=1, \cdots, M) ; \epsilon$ is a small number to avoid denominator 0 during iterative processing.

3.2. Optimization Model with Penalty Term. As it is known, energy consumption of sensor is positively related to distance between sensor and target, at the same time, some sensors die after repeated use, shortening network lifetime. So, it is important that considering the balance of energy consumption to avoid some selected sensors from dying too quickly. Here, we introduce sensor usage factor into problem of sensor selection to constrain energy consumption of selected sensors.

First of all, considering each sensor to be selected at least once for detecting target, we add equation (5) into equation (4) and regard it as the constraint of optimization problem of sensor selection

$$
\sum_{t=1}^{T} \omega_{t} \geq \mathbf{1}
$$

Then, to increase the measurement efficiency, some sensors near the target should be selected with high probability. Therefore, the constraint condition (4) in equation (4) can be modified as follows:

$$
\operatorname{tr}\left(\left(\Phi^{T} \operatorname{diag}(\mathbf{H}) \Phi\right)^{-1}\right) \leq \tau \gamma_{0}-C
$$

where the weight vector of distance is $\mathbf{H}=\left(S_{M} \omega_{t}\right) \odot \mathbf{d}_{M}$, and $\left(\mathbf{d}_{M}\right)_{i}=1 /\left\|\mathbf{m}_{i}-\mathbf{b}\right\|_{2}, S_{M}=S C / M . S_{M}$ is a relative area by monitoring area $S$, environmental factor $C$, and number of sensor nodes $M$.

Next, considering the measurement and processing cost of sensors, we add equation (7) into the constraint condition of equation (4).

$$
(\operatorname{diag}(\mathbf{s}) \Theta \mathbf{1}) \odot \mathbf{P}_{L} \leq \mathbf{e}_{\mathbf{0}}+\mathbf{E},
$$

where $\mathbf{s}=\left[\left\|\varphi_{1}\right\|_{2}^{2},\left\|\varphi_{2}\right\|_{2}^{2}, \cdots,\left\|\varphi_{M}\right\|_{2}^{2}\right]^{T} \in R_{+}^{M}$ is the cost of measurement and data processing about all selected sensors, and $(\mathbf{s})_{i}$ is the cost about the $i$-th sensor. $\left(\mathbf{P}_{L}\right)_{i}=\left(\mathbf{d}_{M}\right)_{i}^{\eta}$ represents the distance-dependent Rayleigh channel attenuation of the $i$ -th sensor, and $\eta$ is the channel attenuation factor. $\mathbf{e}_{0} \in R_{+}^{M}$ is the available reference energy of the sensor, and $\left(\mathbf{e}_{\mathbf{0}}\right)_{i}$ is the energy about the $i$-th sensor; besides, the extra energy beyond the reference energy is expressed by $\mathbf{E} \in R^{M}$.

Obviously, to avoid excessive energy consumption of sensors, it is necessary to add a penalty term $g(\mathbf{E})$ relating to the extra energy in equation (4); therefore, it is revised as follows: 
Input: initialization parameters.

Output: sensor scheduling matrix $\Theta \in\{0,1\}^{M \times T}$.

1: Setting initial weight $\alpha_{t}=\mathbf{1}$, sensor scheduling matrix $\Theta_{0}=\mathbf{0}_{M \times T}$.

2: Initializing unselected sensor node set $N=\varnothing$, and selected node set $K=\varnothing$.

3: Calculating minimum MSE $\gamma_{0}$.

4: FOR st: $=1$ to Maxst DO

5: Obtaining current $\Theta$ by solving optimization problem (14) based on $\Theta_{0}$ Maxst.

6: Updating set $N=\{n \mid \Theta(n) \leq \epsilon\}$ and $K=\{k \mid \Theta(k) \geq 1-\epsilon\}$.

7: $\quad$ IF $\left\|\Theta-\Theta_{0}\right\|_{F}^{2} \leq \epsilon$ THEN

8: $\quad K \longleftarrow K \cup\{\arg \max \Theta(k), k \notin K\}$.

9: $\quad$ ELSE IF $|\mathbf{N}|+|\mathbf{K}| \stackrel{k}{=} M \times T$ THEN

10: $\quad$ Going to step 15.

11: ELSE

12: Setting sensor scheduling matrix $\Theta_{0} \longleftarrow \Theta$, and updating weights based on $\left(\alpha_{t}\right)_{i} \longleftarrow\left(\left(\omega_{t}\right)_{i}+\epsilon\right)^{-1}$, then going to step 5 .

13: END IF

14: END FOR

15: Getting sensor scheduling matrix $\Theta$.

Algorithm 1: The pseudocode of proposed algorithm.

$$
\begin{array}{cc}
\underset{\mathbf{E}>\mathbf{0}, \Theta \in\{0,1\}^{M \times T}}{\operatorname{minimize}} & \sum_{t=1}^{T} \alpha_{t}^{T} \omega_{t}+\lambda g(\mathbf{E}), \\
\text { s.t. } & \operatorname{tr}\left(\left(\Phi^{T} \operatorname{diag}(\mathbf{H}) \Phi\right)^{-1}\right) \leq \tau \gamma_{0}-C, \\
C-\gamma_{0}(\tau-1) \leq 0, & (\operatorname{diag}(\mathbf{s}) \Theta \mathbf{1}) \odot \mathbf{P}_{L} \leq \mathbf{e}_{\mathbf{0}}+\mathbf{E}, \\
\sum_{t=1}^{T} \omega_{t} \geq \mathbf{1},
\end{array}
$$

where penalty term $g(\mathbf{E})=\|\mathbf{E}\|_{2}^{2}, \lambda$ is a regularization coefficient.

3.3. Sensor Selection considering Uncertainty Disturbance. Various uncertainty disturbances may refluent the measurement performance and position of the sensor; thus, we assume that the upper bounds of measurement disturbance and the channel attenuation disturbance are $\delta_{\Phi}$ and $\delta_{P L}$, respectively. Based on the above upper bounds, equation (8) can be transformed as follows:

$$
\begin{gathered}
\underset{\substack{\mathbf{E}>\mathbf{0}, \Theta \in\{0,1\}^{M \times T} \\
\left\|\Delta_{\Phi}\right\|_{2}<\delta_{\Phi},\left\|\Delta_{P L}\right\|_{\infty}<\delta_{p L}}}{\operatorname{minimize}} \sum_{t=1}^{T} \alpha_{t}^{T} \omega_{t}+\lambda g(\mathbf{E}) \\
\text { s.t. } \quad \operatorname{tr}\left(\left(\left(\Phi+\Delta_{\Phi}\right)^{T} \operatorname{diag}(\mathbf{H})\left(\Phi+\Delta_{\Phi}\right)\right)^{-1}\right) \leq \tau \gamma_{0}-C, \\
\\
C-\gamma_{0}(\tau-1) \leq 0 \\
\\
(\operatorname{diag}(\mathbf{s}) \Theta \mathbf{1}) \odot\left(\mathbf{P}_{L}+\Delta_{P L}\right) \leq \mathbf{e}_{\mathbf{0}}+\mathbf{E}, \\
\sum_{t=1}^{T} \omega_{t} \geq \mathbf{1}
\end{gathered}
$$

where $\Delta_{\Phi} \in R^{M \times N}$ and $\Delta_{P L} \in R^{M \times 1}$ are the change of measurement disturbance and channel attenuation disturbance, respectively.

Obviously, the $0-1$ constraint $\Theta \in\{0,1\}^{M \times T}$ is nonconvex; thus, it is necessary to relax the $0-1$ constraint to a polyhedral constraint $\Theta \in[0,1]^{M \times T}$, and equation (9) is rewritten as follows:

$$
\begin{gathered}
\underset{\substack{\mathbf{E}>\mathbf{0}, \Theta \in[0,1]^{M \times T} \\
\left\|\Delta_{\Phi}\right\|_{2}<\delta_{\Phi},\left\|\Delta_{P L}\right\|_{\infty}<\delta_{P L}}}{\operatorname{minimize}} \sum_{t=1}^{T} \alpha_{t}^{T} \omega_{t}+\lambda g(\mathbf{E}), \\
\text { s.t. } \quad \operatorname{tr}\left(\left(\left(\Phi+\Delta_{\Phi}\right)^{T} \operatorname{diag}(\mathbf{H})\left(\Phi+\Delta_{\Phi}\right)\right)^{-1}\right) \leq \tau \gamma_{0}-C, \\
C-\gamma_{0}(\tau-1) \leq 0 \\
(\operatorname{diag}(\mathbf{s}) \Theta \mathbf{1}) \odot\left(\mathbf{P}_{L}+\Delta_{P L}\right) \leq \mathbf{e}_{\mathbf{0}}+\mathbf{E} \\
\sum_{t=1}^{T} \omega_{t} \geq \mathbf{1}
\end{gathered}
$$

where constraint conditions $\left\|\Delta_{\Phi}\right\|_{\infty}<\delta_{\Phi}$ and (10) can be transformed into linear matrix inequality form through matrix transformation as follows (see Appendix A):

$$
\begin{gathered}
\operatorname{tr}\left(\mathbf{U}^{-1}\right) \leq \tau \gamma_{0}-C \\
{\left[\begin{array}{cc}
\Phi^{T} \operatorname{diag}(\mathbf{H}) \Phi-\mathbf{U}-\beta I & \Phi^{T} \operatorname{diag}(\mathbf{H}) \\
\operatorname{diag}(\mathbf{H}) \Phi & \operatorname{diag}(\mathbf{H})+\left(\frac{\beta}{\delta_{\Phi}^{2}}\right) \mathbf{I}
\end{array}\right] \geqslant 0, \quad \beta \geq 0 .}
\end{gathered}
$$

Besides, constraints $\left\|\Delta_{P L}\right\|_{\infty}<\delta_{P L}$ and (10) can also be 
TABLe 2: Parameters of simulation.

\begin{tabular}{|c|c|c|c|c|}
\hline \multirow{2}{*}{ Name } & \multirow{2}{*}{ Symbol } & \multicolumn{3}{|c|}{ Value } \\
\hline & & Scene 1 & Scene 2 & Scene 3 \\
\hline Area & $S$ & $40 \mathrm{~m} \times 40 \mathrm{~m}$ & $70 \mathrm{~m} \times 70 \mathrm{~m}$ & $100 \mathrm{~m} \times 100 \mathrm{~m}$ \\
\hline Number of sensors & $M$ & 100 & 150 & 200 \\
\hline Dimensional of target state & $N$ & 20 & 30 & 40 \\
\hline Position of target & {$\left[b_{x}, b_{y}\right]$} & $(20,20)$ & $(35,35)$ & $(50,50)$ \\
\hline Uncertainty bound of sensor position & $\delta_{P L}$ & 0.8 & 1.6 & 2.4 \\
\hline Level of accuracy of estimation & $\tau$ & & 4 & \\
\hline Attenuation factor of channel & $\eta$ & & -0.5 & \\
\hline Regularization parameter & $\lambda$ & & 1000 & \\
\hline Environmental factor & $\mu$ & & 100 & \\
\hline Relative humidity & $h$ & & $50 \%$ & \\
\hline Temperature & $t_{0}$ & & $25^{\circ} \mathrm{C}$ & \\
\hline Visibility & $V$ & & $500 \mathrm{~m}$ & \\
\hline Measure matrix & $\alpha$ & & 100 & \\
\hline Energy of sensors & $e_{0}$ & & $0 \mathrm{~J}$ & \\
\hline Environmental regulation parameter & $p$ & & $100 \mathrm{~m} /{ }^{\circ} \mathrm{C}$ & \\
\hline
\end{tabular}

transformed into vector form as follows (see Appendix B):

$$
(\operatorname{diag}(\mathbf{s}) \Theta \mathbf{1}) \odot\left(\mathbf{P}_{L}+\Pi\right) \leq \mathbf{e}_{\mathbf{0}}+\mathbf{E},
$$

where

$$
\Pi=[\underbrace{\delta_{P L}, \delta_{P L}, \cdots, \delta_{P L}}_{M}]^{T} .
$$

3.4. Optimization of Sensor Selection. Based on the equations (11) and (12), equation (10) can be rewritten as follows:

$$
\begin{gathered}
\text { s.t. }\left[\begin{array}{cc}
\Phi_{\mathbf{E}>0, \Theta \in[0,1]^{M \times T}}^{T} \operatorname{diag}(\mathbf{H}) \Phi-\mathbf{U}-\beta I & \Phi^{T} \operatorname{diag}(\mathbf{H}) \\
\operatorname{diag}(\mathbf{H}) \Phi & \operatorname{diag}(\mathbf{H})+\left(\frac{\beta}{\delta_{\Phi}^{2}}\right) \mathbf{I}
\end{array}\right] \geqslant 0, \\
(\operatorname{diag}(\mathbf{s}) \Theta \mathbf{1}) \odot\left(\mathbf{P}_{L}+\Pi\right) \leq \mathbf{e}_{\mathbf{0}}+\mathbf{E}, \\
\operatorname{tr}\left(\mathbf{U}^{-1}\right) \leq \tau \gamma_{0}-C, \\
\beta \geq 0, \\
C-\gamma_{0}(\tau-1) \leq 0, \\
\sum_{t=1}^{T} \omega_{t} \geq \mathbf{1} .
\end{gathered}
$$

Obviously, equation (14) is a standard convex optimization problem of sensor selection, and the relaxed sensor scheduling matrix $\Theta$ can be obtained by solving this optimization problem.

Of course, the solution of the relaxed equation (14) cannot directly extend to the original equation (9); thus, we handle this problem by the discretization and legalization method [13].

3.5. Description of the Proposed Algorithm. Algorithm 1 shows the proposed algorithm of sensor selection.

\section{Simulation Results and Analysis}

To test the performance of the proposed algorithm SSCEED, we compare it with the existing algorithms SpareSense [5], FrameSense [6], CON-REL [10], and SS-TECC [11] in a variety of settings as detailed next. We implement all algorithms in MATLAB and the SDP relaxation scheme via CVX [14]. Besides, all simulations are run on a computer with $1.8 \mathrm{GHz}$ Intel Core i5-8250U CPU and 8.00 GB RAM. We give simulation results and analysis on the distribution and number of sensor node selection, besides the estimation accuracy of algorithms with or without disturbance, which are used to describe the performance of the proposed algorithm.

We assume that $M$ stationary sensor nodes carrying the same energy and one target are randomly deployed in simulation scenes 1,2 , and 3 . The parameters with respect to simulations are shown in Table 2; at the same time, for the measurement matrix $\Phi$ with constraint $\Phi^{T} \Phi=\alpha \mathbf{I}$, the selection threshold corresponding to the convex relaxation is set to 0.35 [11].

Figure 1 shows five distributions of sensors and target for the four algorithms in scene 1. 


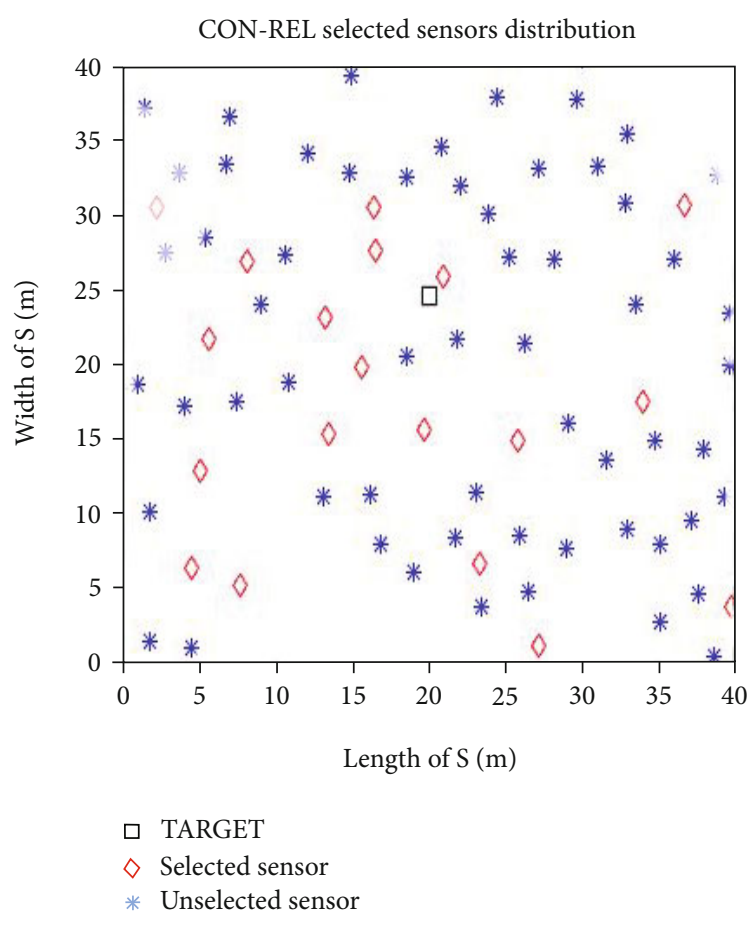

(a) CON-REL

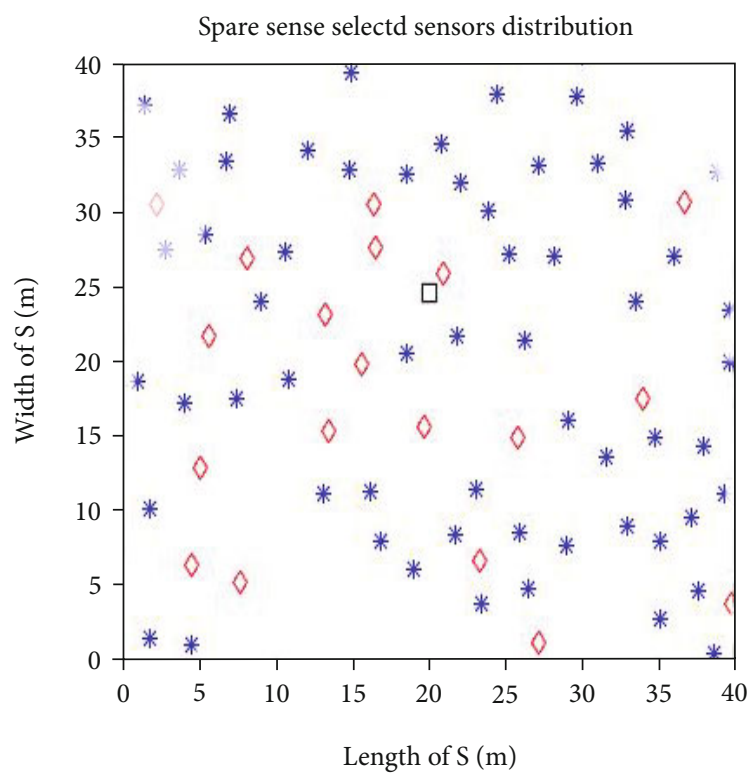

口 TARGET

$\diamond$ Selected sensor

* Unselected sensor

(c) SpareSense

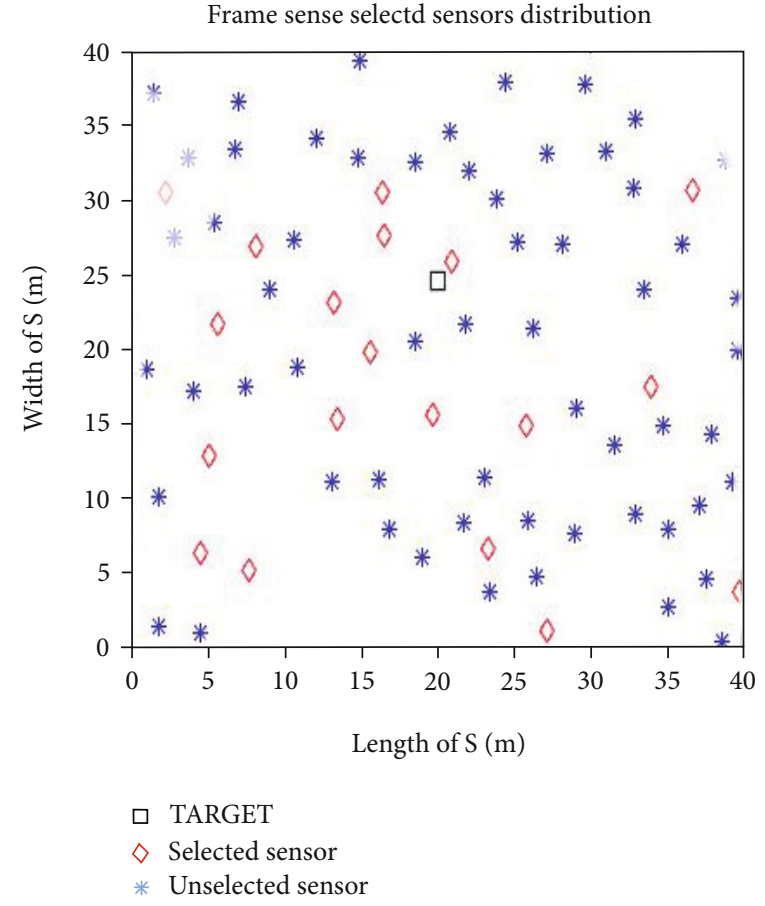

(b) FrameSense

SS-TECC sense selectd sensors distribution

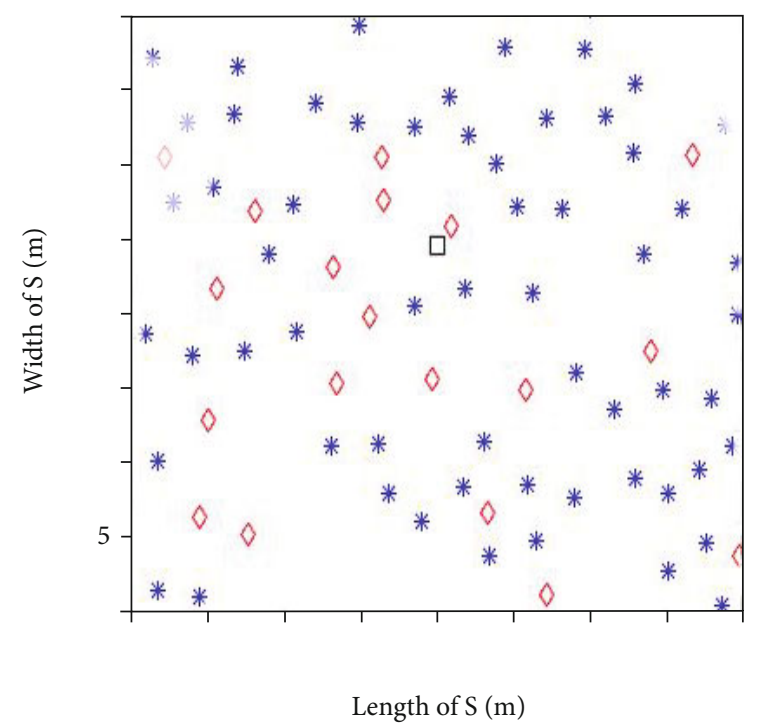

$\square$ TARGET

$\diamond$ Selected sensor

* Unselected sensor

(d) SS-TECC

Figure 1: Continued. 


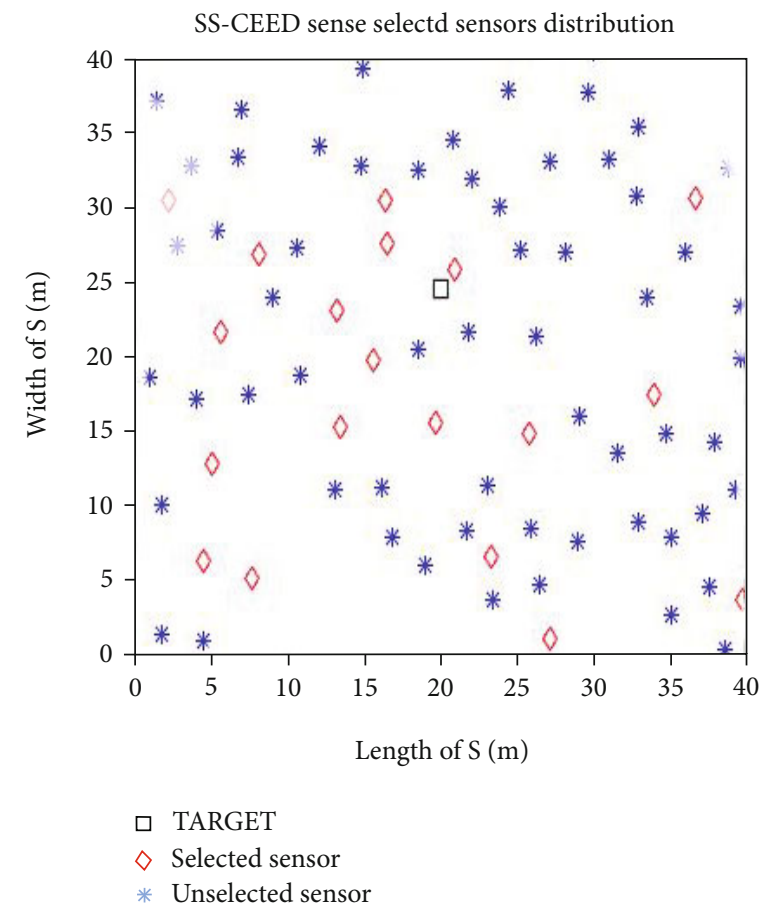

(e) SS-CEED

FIGURE 1: Sensor distribution of five algorithms.

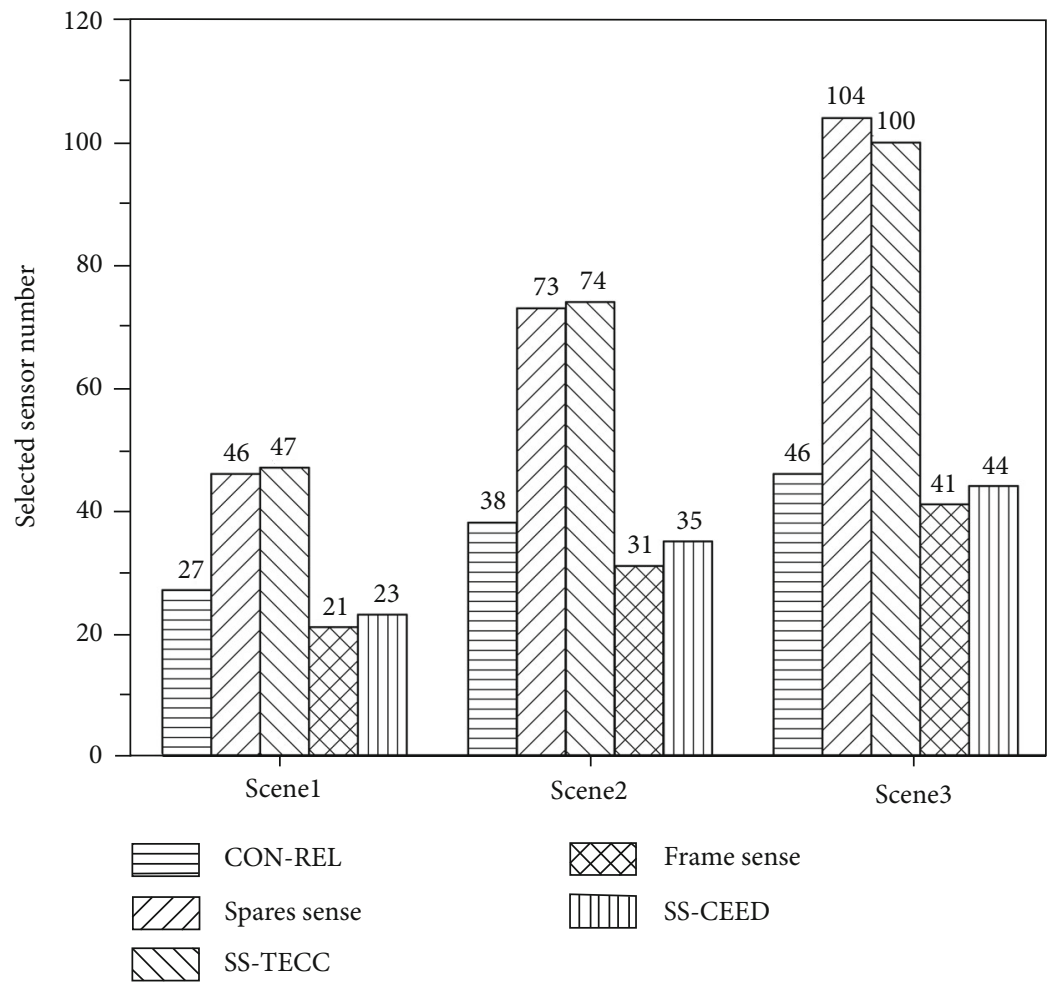

Figure 2: Node selection of algorithms under different scenes.

In Figure 1, symbols “ $\square$," “ $\diamond$," and “*” represent the target, working sensor nodes, and nonworking sensor nodes, respectively. The number of nodes selected by CON-REL, FrameSense, SpareSense, SS-TECC, and SS-CEED is 24, 21,
39,48 , and 21 , respectively. It can be found that the least sensor nodes are selected by the proposed SS-CEED algorithm and the FrameSense algorithm, and the number of selected nodes is greater than the dimension of target $N$ 


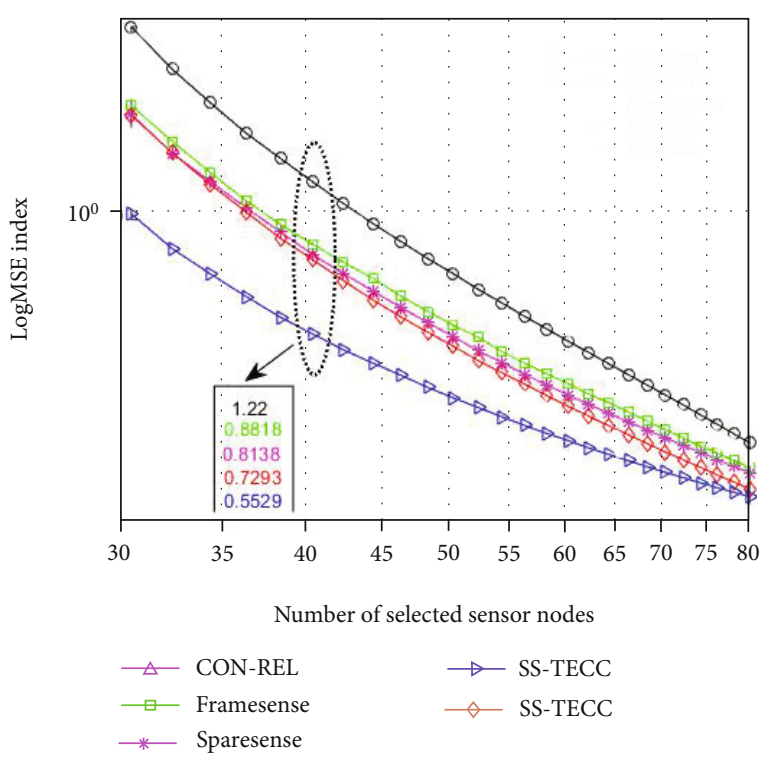

Figure 3: MSE comparison of state estimation.

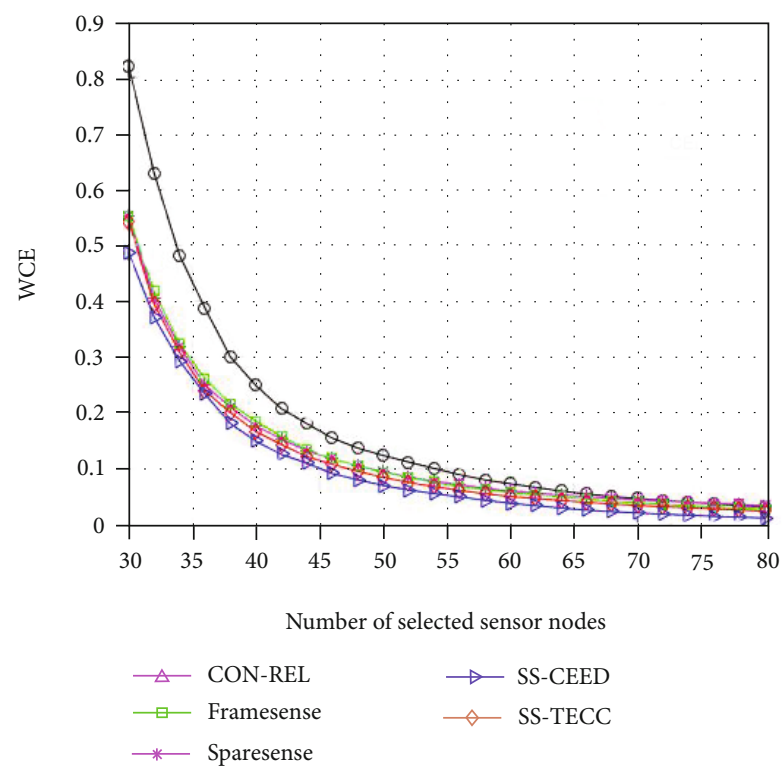

FIGURE 4: WCE comparison of algorithms.

$(N=20)$.The reason is that the FrameSense algorithm excludes one node at every time of selecting the senor until the remaining number is $N+1$, i.e., there have 21 sensors at this time. Other algorithms are similar in selecting sensor nodes. Besides, compared with other algorithms, distance weight is considered in the SS-CEED algorithm, leading to the sensor nodes nearby the target being selected to work; it can effectively decrease energy consumption of communication.

4.1. Analysis of Node Selection. To clearly describe the algorithm performance in different scenes, Figure 2 gives the average number of selected sensors by 100 times Monte Carlo simulation.

In Figure 2, the five algorithms' number of selected sensor nodes increases when the number of deployed nodes increases

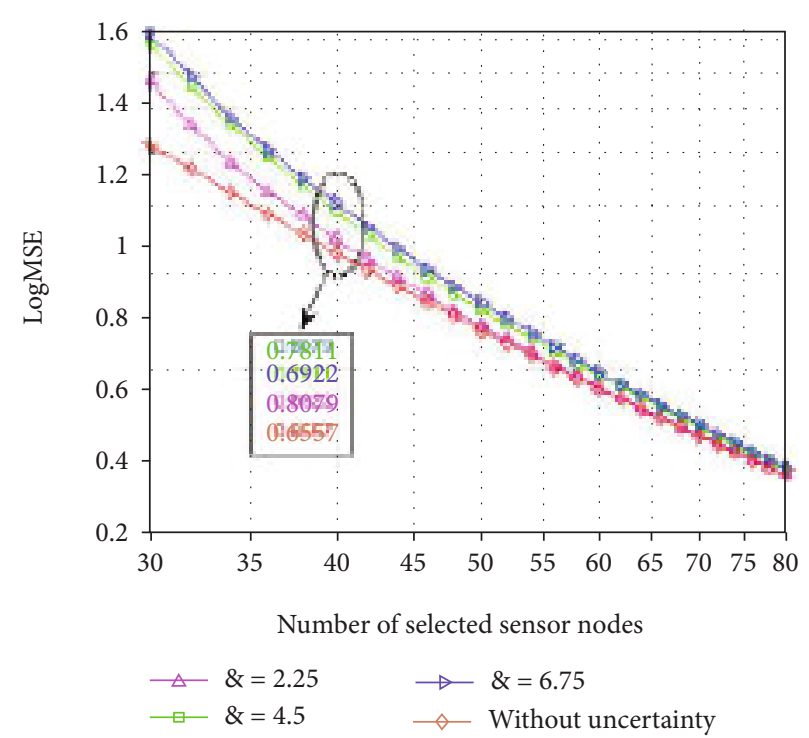

FIgURE 5: The logMSE comparison under different measurement disturbances.

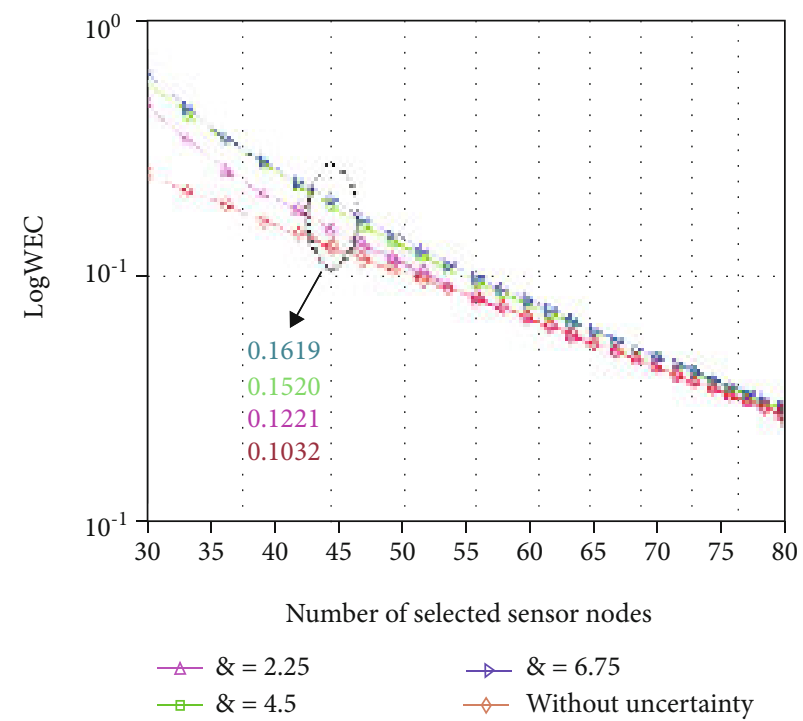

FIgURE 6: The logWCE comparison under different measurement disturbances.

in different scenes. The proposed SS-CEED algorithm selects the sensor number as 23, 35, and 44 under three scenes. Compared with other algorithms excluding FrameSense, selecting the sensor number of the proposed SS-CEED algorithm is minimum, and this conclusion is consistent with the analysis result in Figure 1. Although the FrameSense algorithm can select the least number of sensors, it requires that the number of selected sensor nodes must be one more than the dimension of sensor measurement; however, the above constraint is not necessary for the SS-CEED algorithm.

4.2. Analysis of Estimation Error. To show selection performance of five algorithms, this paper gives MSE of target state estimation after deploying selected sensors under different 


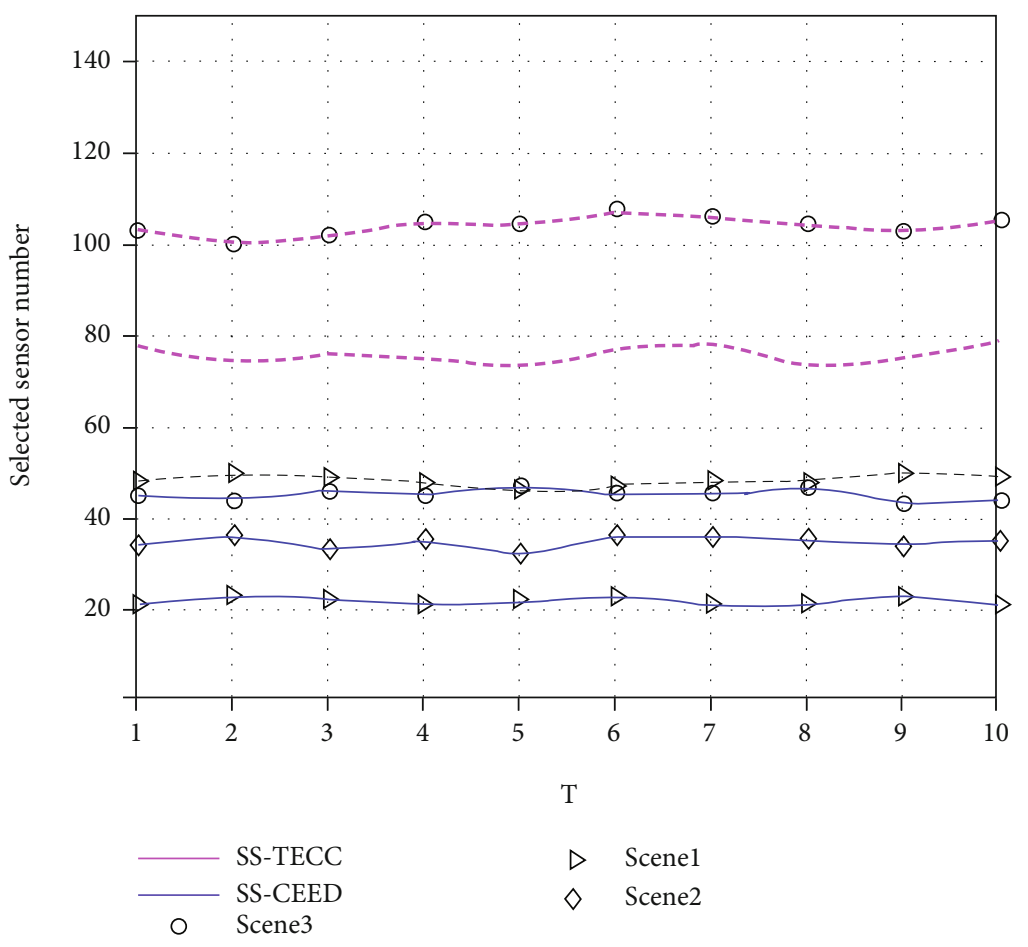

FIgURE 7: Comparison of the number of selected sensors in different scenes.

algorithms. Regarding scene 1 as the simulation background, we compare the MSE and WCE of algorithms under measurement bounded disturbance $\delta_{\Phi}=1.2$. Figures 3 and 4 give MSE and WCE of algorithms under different selected sensor numbers, respectively.

The logarithm of MSE (logMSE) is displayed to clearly describe the estimation error in Figure 3. It is found that all $\log$ MSEs are decreased when the number of selected sensor nodes increase. The reason is that estimation accuracy improves and MSE decreases with the number of sensor nodes used for state estimation increases. In this figure, the logMSE of the proposed SS-CEED algorithm has the smallest value of 0.5529 when the number of selected sensors is 40 , and it is $0.1764,0.2609,0.3289$, and 0.6671 lower than that of SS-TECC, SpareSense, FrameSense, and CON-REL, respectively. The reason is that the SS-CEED algorithm considers measurement and position disturbance of sensors, leading to its estimation performance being the best.

Similar to Figure 3, all WCEs are decreased when the number of selected sensor nodes increases in Figure 4. Compared with SS-TECC, SpareSense, FrameSense, and CONREL, the proposed SS-CEED algorithm has the smallest WCE, meaning accuracy of state estimation is best.

4.3. Performance Analysis under Different Disturbances. To analyze the influence of different measurement disturbances on the estimation accuracy of the SS-CEED algorithm, this paper gives the result of MSE and WCE after 100 times Monte Carlo simulation under scene 1. Similar to Figure 3, in order to clearly describe the results, the logarithms of MSE and WCE are shown in Figures 5 and 6, named as logMSE and logWCE, respectively.
Assuming the bound measurement disturbances are 0, $2.25,4.5$, and 6.75 , Figure 5 shows the logMSE curve of the proposed algorithm. The logMSE of the proposed algorithm is the lowest without measurement disturbance. When there is measurement disturbance, taking 40 sensor nodes as an example, the logMSE with disturbance boundary of 2.25 is 0.0889 smaller than the disturbance boundary of 4.5 and is 0.1157 smaller than the disturbance boundary of 6.75 . It is found from different curves of selected sensor nodes. In short, considering measurement disturbance, the estimation accuracy of the proposed algorithm decreases as the measurement disturbance boundary increases.

Similar to logMSE in Figure 5, the logWCE of the proposed algorithm is the lowest without measurement disturbance in Figure 6. And taking 40 sensors as an example, the $\log$ WCE with a disturbance boundary of 2.25 is 0.0299 smaller than the disturbance boundary of 4.5 and is 0.0398 smaller than the disturbance boundary of 6.75. Similarly, the estimation accuracy of the proposed algorithm decreases as the measurement disturbance boundary increases under considering measurement disturbance.

4.4. Analysis of Sensor Node Selection in Different Times. Different from other algorithms, the SS-TECC algorithm and the proposed SS-CEED algorithm involve sensor node selection in different working times. In Figure 7, comparison of selection number between the SS-TECC algorithm and the SS-CEED algorithm under all scenes is within $T=10$ times.

It can be seen from Figure 7, in observation period of the three scenarios, the number of sensors selected by the SSCEED algorithm and the SS-TECC algorithm changes little; that is, the number of sensor nodes selected by the two 
algorithms changes within 5 to save energy. It is obvious that the number of sensors selected by the SS-CEED algorithm is less than that by the SS-TECC algorithm, and the sensor number variation of the SS-CEED algorithm is stationary. In short, compared with the SS-TECC algorithm, the SSCEED algorithm selects fewer sensors nodes, which can save more network energy.

\section{Conclusions}

Random disturbances, energy carried by the sensor, distance between the target and the sensor node, and so on affect the performance of sensor selection, so this paper regards the sensor selection problem as a binary optimization problem for sensor scheduling to effectively select sensors to deploy. The optimization problem aimed at the minimum number of selected sensor nodes is constructed, and the constraints such as energy consumption and precision are given. Using matrix transformation and convex relaxation techniques, the optimization problem is solved, and the sensor selection scheme is given. The proposed algorithm not only meets the target state estimation accuracy but also effectively reduces sensor resources and network energy consumption.

\section{Appendix}

\section{A. Transformation of Matrix Inequality (11)}

Setting $\bar{\Phi}=\Phi+\Delta_{\Phi},\left\|\Delta_{\Phi}\right\|_{2}<\delta_{\Phi}$, then the optimization constraint (10) can be expressed as

$$
\begin{gathered}
\operatorname{tr}\left(\mathbf{U}^{-1}\right) \leq \tau \gamma_{0}-C, \\
\mathbf{U} \leq \bar{\Phi}^{\mathrm{T}} \operatorname{diag}(\mathbf{H}) \bar{\Phi}, \\
\left\|\Delta_{\Phi}\right\|_{2}<\delta_{\Phi},
\end{gathered}
$$

where $\mathbf{U} \in \mathbf{R}^{N \times N}$ is a symmetric positive definite matrix.

Assuming $\Delta_{\Phi}$ is bounded, the equation (11) can be transformed into linear matrix inequality. The proof is as follows.

First, the constraint $\left\|\Delta_{\Phi}\right\|_{2} \leq \delta_{\Phi}$ is transformed into $\Delta_{\Phi}{ }^{T} \Delta_{\Phi} \preceq \delta_{\Phi}{ }^{2} I$, then $\bar{\Phi}=\Phi+\Delta_{\Phi}$ is substituted into $\mathbf{U} \preceq \bar{\Phi}^{T}$ $\operatorname{diag}(\mathbf{H}) \bar{\Phi}$, and inequality (A.2) is received

$$
\begin{gathered}
\mathbf{U} \preceq \Phi^{T} \operatorname{diag}(\mathbf{H}) \Phi+\Phi^{T} \operatorname{diag}(\mathbf{H}) \Delta_{\Phi} \\
+\Delta_{\Phi}{ }^{T} \operatorname{diag}(\mathbf{H}) \Phi+\Delta_{\Phi}{ }^{T} \operatorname{diag}(\mathbf{H}) \Delta_{\Phi}, \\
\Delta_{\Phi}{ }^{T} \Delta_{\Phi} \preceq \delta_{\Phi}{ }^{2} I .
\end{gathered}
$$

Theorem 3.3 in [15] shows that the above semi-infinite quadratic matrix inequality holds if and only if the following matrix inequality (A.3) holds.

$$
\left[\begin{array}{cc}
\Phi^{T} \operatorname{diag}(\mathbf{H}) \Phi-\mathbf{U}-\beta I & \Phi^{T} \operatorname{diag}(\mathbf{H}) \\
\operatorname{diag}(\mathbf{H}) \Phi & \operatorname{diag}(\mathbf{H})+\left(\frac{\beta}{{\delta_{\Phi}^{2}}^{2}}\right) \mathbf{I}
\end{array}\right] \geqslant 0, \quad \beta \geq 0 .
$$

Thus, the equation (A.1) can be converted into the following inequality:

$$
\begin{gathered}
\operatorname{tr}\left(\mathbf{U}^{-1}\right) \leq \tau \gamma_{0}-C, \\
{\left[\begin{array}{cc}
\Phi^{T} \operatorname{diag}(\mathbf{H}) \Phi-\mathbf{U}-\beta I & \Phi^{T} \operatorname{diag}(\mathbf{H}) \\
\operatorname{diag}(\mathbf{H}) \Phi & \operatorname{diag}(\mathbf{H})+\left(\frac{\beta}{\delta_{\Phi}^{2}}\right) \mathbf{I}
\end{array}\right] \geqslant 0, \quad \beta \geq 0 .}
\end{gathered}
$$

\section{B. Transformation of Inequality (12)}

Setting $\overline{\mathbf{P}}_{L}=\mathbf{P}_{L}+\Delta_{P L},\left\|\Delta_{P L}\right\|_{\infty}<\delta_{P L}$, then the optimization constraint (10) can be expressed as

$\sup \left\{(\operatorname{diag}(\mathbf{s}) \Theta \mathbf{1}) \odot \overline{\mathbf{P}}_{L}\right\}_{i} \leq\left(\mathbf{e}_{\mathbf{0}}+\mathbf{E}\right)_{i} \quad\left\|\Delta_{P L}\right\|_{\infty}<\delta_{P L}, \quad i=1, \cdots, M$.

The left-hand side of equation (B.1) can be expressed as

$$
\begin{aligned}
\sup & \left\{\left((\operatorname{diag}(\mathbf{s}) \Theta \mathbf{1}) \odot \overline{\mathbf{P}}_{L}\right)_{i}\right\} \\
= & \left(\operatorname{diag}(\mathbf{s}) \Theta \mathbf{1} \odot \mathbf{P}_{L}\right)_{i}+\sup \left\{(\operatorname{diag}(\mathbf{s}) \Theta \mathbf{1}) \odot\left(\Delta_{P L}\right)_{i}\right\} \quad i=1, \cdots, M \\
= & \left(\operatorname{diag}(\mathbf{s}) \Theta \mathbf{1} \odot \mathbf{P}_{L}\right)_{i}+\delta_{P L}(\operatorname{diag}(\mathbf{s}) \Theta \mathbf{1})_{i} \quad i=1, \cdots, M .
\end{aligned}
$$

Making

$$
\Pi=[\underbrace{\delta_{P L}, \delta_{P L}, \cdots, \delta_{P L}}_{M}]^{T},
$$

we can vectorize the scalar representation and get the following inequality:

$$
(\operatorname{diag}(\mathbf{s}) \Theta \mathbf{1}) \odot\left(\mathbf{P}_{L}+\Pi\right) \leq \mathbf{e}_{\mathbf{0}}+\mathbf{E}
$$

\section{Data Availability}

For simulation data, please contact the corresponding author of this manuscript.

\section{Conflicts of Interest}

The authors declare that they have no conflicts of interest.

\section{Acknowledgments}

This work was supported by the National Science Foundation Council of China (61771006, 61976080); the Key Research Projects of University in Henan Province, China (21A413002, 19A413006, 20B510001); the Programs for Science and Technology Development of Henan Province, China (192102210254); and the Soft Science Research Program of Henan Province, China (202400410097). 


\section{References}

[1] B. Wang, J. Zhu, L. T. Yang, and Y. Mo, "Sensor density for confident information coverage in randomly deployed sensor networks," IEEE Transactions on Wireless Communications, vol. 15, no. 5, pp. 3238-3250, 2016.

[2] V. Roy, A. Simonetto, and G. Leus, "Spatio-temporal sensor management for environmental field estimation," Signal Processing, vol. 128, pp. 369-381, 2016.

[3] X. Wang, X. Liu, C.-T. Cheng, L. Deng, X. Chen, and F. Xiao, "A joint user scheduling and trajectory planning data collection strategy for the UAV-assisted WSN," IEEE Communications Letters, vol. 25, no. 7, pp. 2333-2337, 2021.

[4] M. Hibbard, K. Tuggle, and T. Tanaka, "Point-based value iteration and approximately optimal dynamic sensor selection for linear-Gaussian processes," IEEE Control Systems Letters, vol. 5, no. 6, pp. 2192-2197, 2021.

[5] H. Jamali, A. Simonetto, and G. Leus, "Sparsity-aware sensor selection: centralized and distributed algorithms," IEEE Signal Processing Letters, vol. 21, no. 2, pp. 217-220, 2014.

[6] J. Ranieri, A. Chebira, and M. Vetterli, "Near-optimal sensor placement for linear inverse problems," IEEE Transactions on Signal Processing, vol. 62, no. 5, pp. 1135-1146, 2014.

[7] C. Jiang, Y. C. Soh, and H. Li, "Sensor placement by maximal projection on minimum eigenspace for linear inverse problems," IEEE Transactions on Signal Processing, vol. 64, no. 21, pp. 5595-5610, 2016.

[8] C. Jiang, Z. Chen, R. Su, and Y. C. Soh, "Group greedy method for sensor placement," IEEE Transactions on Signal Processing, vol. 67, no. 9, pp. 2249-2262, 2019.

[9] J. Luo and P. Pattipati, "Fast optimal and suboptimal any-time algorithms for CDMA multiuser detection based on branch and bound," IEEE Transactions on Communications, vol. 52, no. 4, pp. 632-642, 2004.

[10] S. Joshi and S. Boyd, "Sensor selection via convex optimization," IEEE Transactions on Signal Processing, vol. 57, no. 2, pp. 451-462, 2009.

[11] C. Rusu, J. Thompson, and N. M. Robertson, "Sensor scheduling with time, energy, and communication constraints," IEEE Transactions on Signal Processing, vol. 66, no. 2, pp. 528-539, 2018.

[12] S. K. Sengijpta, "Fundamentals of statistical signal processing: estimation theory," Control Engineering Practice, vol. 37, no. 4, pp. 465-466, 1994.

[13] G. Xu, C. Pang, and X. Duan, "Multi-sensor optimization scheduling for target tracking based on PCRLB and a novel intercept probability factor," Electronics, vol. 8, no. 2, pp. 140-162, 2019.

[14] M. Grant, S. Boyd, and Y. Ye, "CVX: Matlab software for disciplined convex programming," in Book Series Nonconvex Optimization and Its Applications, pp. 155-210, Springer, 2006.

[15] A. Mutapcic and S. Boyd, "Cutting-set methods for robust convex optimization with pessimizing oracles," Optimization Methods and Software, vol. 24, no. 3, pp. 381-406, 2009. 